\title{
Optimal Decoding for Hard-Decision Forwarding Aided Cooperative Spatial Multiplexing Systems
}

\author{
Kyungchun Lee and Lajos Hanzo
}

\begin{abstract}
In this letter, the optimal decoding strategy for cooperative spatial multiplexing (CSM) aided systems is derived. In CSM systems, the multiple relay stations (RSs), which compose a virtual antenna array (VAA), independently decode the packets received from the mobile stations (MS) and forward them to the base station (BS). When the BS decodes the signal forwarded from the RSs, the potential decoding errors encountered at the RSs will result in erroneous forwarding, but their effects are mitigated by the proposed solution. Our simulation results show that when the relay link has a significantly higher signal-tonoise ratio than the direct link, the proposed decoding algorithm achieves an approximately $3 \mathrm{~dB}$ better performance than conventional CSM, which does not consider the deleterious effects of erroneous forwarding from the RSs.
\end{abstract}

Index Terms-Cooperative communication, virtual array antenna, multiple-input multiple-output (MIMO) system.

\section{INTRODUCTION}

$\mathbf{M}$ ULTIPLE-INPUT multiple-output (MIMO) systems are capable of dramatically increasing the channel capacity, which grows linearly with the number of transmit or receive antennas, whichever is lower [1]-[3]. However, the mobile stations (MSs) have a limited-size and hence they are typically equipped with a single antenna, which erodes the achievable capacity gain of MIMO systems. To mitigate this problem, virtual antenna arrays (VAAs) based on cooperative and relay station (RS)-aided communications have been studied [5]-[11]. In the uplink (UL) of VAA-aided systems, the source information is first transmitted from the MS to multiple RSs, and then the RSs simultaneously retransmit the received packet to the base station (BS) having multiple receive antennas. By composing a virtual MIMO channel between the multiple RSs and the BS, substantial diversity and multiplexing gains can be achieved. Furthermore, since the RSs are typically far apart, the signals transmitted from the RSs experience independent fading, which ensures a higher diversity gain [4], [5]. By using VAAs based on multiple RSs, the effects of shadow fading can also be mitigated. The RSs may be constituted by the MSs, which are currently not engaged in active communication with the BS, but fixed RSs may also be installed.

Manuscript received June 16, 2008; revised January 13, 2009 and May 8, 2009; accepted May 8, 2009. The associate editor coordinating the review of this letter and approving it for publication was I. Collings.

This work was supported by the Korea Research Foundation Grant funded by the Korean Government (MOEHRD) (KRF-2007-357-D00110).

K. Lee is with Samsung Electronics, P.O. Box 105, Suwon, Korea (e-mail: kclee@sclab.kaist.ac.kr).

L. Hanzo is with the School of Electronics and Computer Science, University of Southampton, SO17 1BJ Southampton, U.K. (e-mail: $1 \mathrm{~h} @$ ecs.soton.ac.uk).

Digital Object Identifier 10.1109/TWC.2009.080804
Similar to collocated antenna array, various MIMO transmission modes can be applied to the VAAs, such as for example, space-time coding (STC) [6]-[8] designed for attaining a high diversity gain. By contrast, cooperative spatial multiplexing (CSM) was contrived to maximise the achievable throughput in multiple RS aided VAAs [7], [9]-[11]. In the CSM transmission mode, the UL packet received at the RSs in a high-rate serial transmission mode is divided into several lower-rate substreams, which are simultaneously transmitted in parallel from the RSs to the BS. More specifically, each RS independently decodes the entire UL packet based on its own received signal, but it forwards only a part of the entire packet using an error-resilient mode without reducing the overall throughput. Compared to classic relaying systems, the CSM arrangement is capable of reducing the required temporal and spectral resources as a benefit of parallel-rather than individual serial-transmission from multiple RSs. Consequently, by employing CSM, rather than a single RS-aided scheme using for example multiple time-slots, the effective throughput can be increased.

In this letter, a novel CSM transmission scheme is proposed. It is typically assumed that RSs perfectly decode the packets received from the source. However, in practice, decoding errors may occur at the RSs and hence the erroneous packets are forwarded from the RSs to the destination, which may substantially affect the attainable end-to-end performance. To circumvent this problem, we develop an efficient decoding algorithm for the destination node (i.e. for the BS in the UL), which is capable of mitigating the associated error propagation. In the proposed algorithm, the decoding errors encountered at the RSs are taken into account, when iterative MIMO detection/decoding is performed at the BS. More explicitly, the decoding errors of a RS can be corrected at the BS with the aid of the side information, which is generated from the substreams received from other RSs as well as from the signal directly received from the source node.

This paper is organized as follows. Section II describes the system model of multiple RS aided CSM communication. In Section III, the proposed decoding algorithm is derived, while Section IV provides our simulation results. In Section V, our conclusions are presented

\section{System Model}

Both the MS and the RSs are assumed to have a single antenna ${ }^{1}$, while the BS has $N_{B}$ receive antennas. We assume

\footnotetext{
${ }^{1}$ In this letter, the proposed algorithm is derived under assumption that the MS and RSs adopt a single antenna. However, the proposed algorithm can be readily extended to the scenario of having multiple antennas at the MS and RSs.
} 


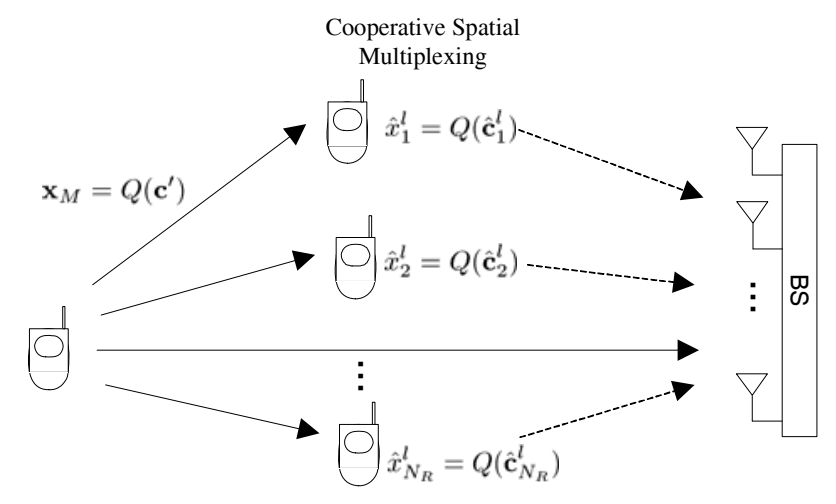

$N_{R}$ RSs

Fig. 1. Cooperative spatial multiplexing systems.

that $N_{R}$ RSs support the UL communication, which results in a $\left(N_{B} \times N_{R}\right)$-dimensional virtual MIMO channel matrix between the RSs and BS. In the first time-slot (TS), the MS encodes the UL information bit stream into a codeword $\mathbf{c}^{\prime}$, which contains $L_{c}$ coded bits. The codeword $\mathbf{c}^{\prime}$ is then mapped to the symbol stream $\mathbf{x}_{M}$, which is the $\left(L_{x} \times 1\right)$ element symbol vector. Then, $\mathbf{x}_{M}$ is transmitted from the MS to the RSs, as shown in Fig. 1. The signal received at the $n$th RS during the $l$ th channel use becomes $y_{R, n}^{l}=$ $h_{R, n} x_{M}^{l}+v_{R, n}^{l}, \quad n=1,2, \cdots, N_{R}, l=1,2, \cdots, L_{x}$, where $x_{M}^{l}$ is the $l$ th element of $\mathbf{x}_{M}$ and $y_{R, n}^{l}$ is the corresponding received signal. Furthermore, $h_{R, n}$ represents the complexvalued channel coefficient and $v_{R, n}^{l}$ indicates the independent zero-mean complex Gaussian noise having a variance of $\sigma_{v}^{2} / 2$ per dimension.

We note that during the first TS, the BS also receives the symbol stream $\mathbf{x}_{M}$, which is directly transmitted from the MS, even though the direct link between the MS and BS typically has a lower signal-to-noise ratio (SNR) than the relay link. The signal received at the BS can be written as

$$
\mathbf{y}_{B}^{l}=\mathbf{h}_{B} x_{M}^{l}+\mathbf{v}_{B}^{l}, \quad l=1,2, \cdots, L_{x},
$$

where $\mathbf{y}_{B}^{l}$ represents the $\left(N_{B} \times 1\right)$-element received signal vector and $\mathbf{h}_{B}$ denotes the $\left(N_{B} \times 1\right)$-element complex-valued channel vector. Furthermore, $\mathbf{v}_{B}^{l}$ indicates the independent zero-mean complex Gaussian noise vector having a variance of $\sigma_{v}^{2} / 2$ per dimension.

Exploiting the signal received from the MS, each RS estimates the codeword $\mathbf{c}^{\prime}$ by performing channel decoding. The estimate of $\mathbf{c}^{\prime}$ at the $n$th RS is denoted as $\hat{\mathbf{c}}_{n}^{\prime}$. Then, $\hat{\mathbf{c}}_{n}^{\prime}$ is forwarded to the interleaver $\boldsymbol{\Pi}_{R}(\cdot)$ in order to obtain $\hat{\mathbf{c}}_{n}=\boldsymbol{\Pi}_{R}\left(\hat{\mathbf{c}}_{n}^{\prime}\right)$. If the received packet is perfectly decoded at each RS, we have $\hat{\mathbf{c}}_{1}=\hat{\mathbf{c}}_{2}=\cdots=\hat{\mathbf{c}}_{N_{R}}=\mathbf{c}$, where $\mathbf{c}=$ $\Pi_{R}\left(\mathbf{c}^{\prime}\right)$. For the sake of benefitting from spatial multiplexing aided transmissions, the interleaved codeword $\hat{\mathbf{c}}_{n}$ is divided into $N_{R}$ reduced-length substreams $\left\{\hat{\mathbf{c}}_{n, 1}, \hat{\mathbf{c}}_{n, 2}, \cdots, \hat{\mathbf{c}}_{n, N_{R}}\right\}$, where $\hat{\mathbf{c}}_{n, k}$ indicates the $k$ th $\left(L_{c} / N_{R}\right)$-length substream of $\hat{\mathbf{c}}_{n}$. Given the $N_{R}$ substreams, the $n$th RS only transmits the $n$th substream $\hat{\mathbf{c}}_{n, n}$ to the BS. More specifically, $N_{R}$ RSs transmit $N_{R}$ substreams in parallel so that the entire codeword of $\mathbf{c}$ is received at the BS during the second TS.

The substream $\hat{\mathbf{c}}_{n, n}$ of the $n$th RS can be expressed as

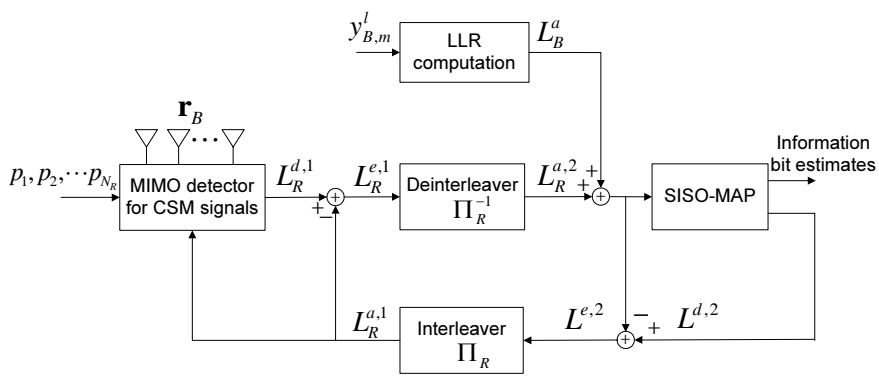

Fig. 2. Block diagram of the proposed decoding scheme.

$\hat{\mathbf{c}}_{n, n}=\left\{\hat{\mathbf{c}}_{n}^{1}, \hat{\mathbf{c}}_{n}^{2}, \cdots, \hat{\mathbf{c}}_{n}^{L_{s} / N_{R}}\right\}$, where $\hat{\mathbf{c}}_{n}^{l}$ is the coded bit vector associated with the symbol $\hat{x}_{n}^{l}$, which is transmitted from the $n$th RS during the $l$ th channel use. The overall received signal at the $\mathrm{BS}$ during the $l$ th channel use of the second TS can be expressed as ${ }^{2}$

$$
\mathbf{r}_{B}=\mathbf{G} \hat{\mathbf{x}}_{R}+\mathbf{v}_{B}
$$

where $\mathbf{r}_{B}$ represents the $\left(N_{B} \times 1\right)$-element complex-valued received signal vector, while $\hat{\mathbf{x}}_{R}=\left[\hat{x}_{1}^{l} \hat{x}_{2}^{l} \cdots \hat{x}_{N_{R}}^{l}\right]^{T}$ indicates the symbol vector transmitted across the RSs during the $l$ th channel use. Furthermore, $\mathbf{G}$ denotes the $\left(N_{B} \times N_{R}\right)$-element complex-valued channel matrix, where $[\mathbf{G}]_{m n}$ indicates the channel coefficient between the $n$th $\mathrm{RS}$ and the $m$ th receive antenna of the BS. Still considering (2), $\mathbf{v}_{B}$ denotes the $\left(N_{B} \times\right.$ 1)-element noise vector of independent zero-mean complex Gaussian entries having a variance of $\sigma_{v}^{2} / 2$ per dimension. It is worth noting that the received signal model of (2) is reminiscent of the vertically encoded MIMO system of [12], [13], which transmits a segment of a single codeword from each transmit antenna.

By applying the now classic iterative detection and decoding (IDD) algorithm to the received signal $\mathbf{r}_{B}$ [12], [13], the BS decodes the UL packet $\mathbf{c}^{\prime}$. As shown in Fig. 2, the directly detected signal $y_{B, m}^{l}$ of (1) can also be exploited in order to improve the attainable performance. More specifically, the log-likelihood ratios (LLRs) of coded bits are computed by the directly detected signal based on the conventional maximum ratio combining reception, and then added to the LLRs computed by the relayed signal in order to obtain a more reliable input of the channel decoder. When decoding $\mathbf{c}^{\prime}$, it is generally assumed that the RSs forwarded perfectly decoded data. However, in practical relaying systems, decoding errors may occur at the RSs, which causes error propagation at the BS. In order to mitigate error propagation, an efficient decoding algorithm will be proposed in the forthcoming section, which considers potentially erroneous signal transmission from the RSs. The proposed algorithm is derived by extending the decoding algorithm of [14] to CSM systems.

\section{Proposed Decoding Algorithm}

The bit error probability (BEP) within the decoded packet $\hat{\mathbf{c}}_{n}$, which is the interleaved version of the channel decoder's output at the $n$th RS, is denoted as $p_{n}(<1 / 2)$, i.e. $p_{n}=d\left(\hat{\mathbf{c}}_{n}, \mathbf{c}\right) / L_{c}$, where $d(\cdot, \cdot)$ represents the Hamming

${ }^{2}$ For notational convenience, we omit the channel use index throughout the paper, when it does not cause any confusion. 
distance between two vectors. Furthermore, we denote the coded bit vector associated with the $l$ th symbol vector $\hat{\mathbf{x}}_{R}$ as $\hat{\mathbf{c}}^{l}=\left\{\hat{\mathbf{c}}_{1}^{l}, \hat{\mathbf{c}}_{2}^{l}, \cdots, \hat{\mathbf{c}}_{N_{R}}^{l}\right\}$. We note that the elements of $\hat{\mathbf{x}}_{R}$ are independently transmitted from the different RSs and $\hat{\mathbf{c}}_{n}^{l}$ is the estimated coded bit stream at the $n$th RS. Then, $\hat{\mathbf{c}}^{l}$ can be written as $\hat{\mathbf{c}}^{l}=\mathbf{c}^{l} \oplus \Delta \mathbf{c}^{l}$, where $\mathbf{c}^{l}$ denotes the corresponding exact coded bits, while $\Delta \mathbf{c}^{l} \in\{0,1\}^{K}$ represents the $K$ element vector of decoding errors in $\hat{\mathbf{c}}^{l}$. Here, $\oplus$ denotes the element-wise XOR operation, while $K$ denotes the size of $\hat{\mathbf{c}}^{l}$, namely the number of coded bits associated with the symbol vector $\hat{\mathbf{x}}_{R}$. Furthermore, the $k$ th bit of $\hat{\mathbf{c}}^{l}$ can be expressed as $\hat{c}_{k}=c_{k} \oplus \Delta c_{k}$, where $c_{k}$ and $\Delta c_{k}$ indicate the $k$ th elements of $\mathbf{c}^{l}$ and $\Delta \mathbf{c}^{l}$, respectively. We express the probability of $\Delta c_{k}$ as

$$
p\left(\Delta c_{k}\right)=\left\{\begin{array}{cc}
1-q_{k}, & \text { if } \Delta c_{k}=0 \\
q_{k}, & \text { if } \Delta c_{k}=1
\end{array},\right.
$$

where $q_{k}$ is equal to one of the $N_{R}$ RS BEPs $\left\{p_{1}, p_{2}, \cdots, p_{N_{R}}\right\}$. More specifically, if $\hat{c}_{k}$ is the coded bit transmitted from the $n$th $\mathrm{RS}$, its error probability becomes $p_{n}$, i.e., $q_{k}=p_{n}$.

The signal directly received from the MS does not contain the RS's decision error hosted by $\Delta \mathbf{c}^{l}$ and hence the conventional channel decoder is applied to $y_{B, m}^{l}$ of (1) without any modification. Therefore, we only have to modify the MIMO detection block of the CSM signals of (2) seen in Fig. 2 by considering the decoding error vector $\Delta \mathbf{c}^{l}$. The LLR of $c_{k}$ is formulated as

$L_{R}^{d, 1}\left(c_{k}\right)=\log \frac{\sum_{\left\{\mathbf{c}^{l}, \Delta \mathbf{c}^{l}\right\}: c_{k}=1} e^{-\left\|\mathbf{r}_{B}-\mathbf{G} \tilde{\mathbf{x}}_{R}\right\|^{2} / \sigma^{2}} p\left(\mathbf{c}^{l}, \Delta \mathbf{c}^{l}\right)}{\sum_{\left\{\mathbf{c}^{l}, \Delta \mathbf{c}^{l}\right\}: c_{k}=0} e^{-\left\|\mathbf{r}_{B}-\mathbf{G} \tilde{\mathbf{x}}_{R}\right\|^{2} / \sigma^{2} p\left(\mathbf{c}^{l}, \Delta \mathbf{c}^{l}\right)}}$,

where $\tilde{\mathbf{x}}_{R}$ is a trial of $\hat{\mathbf{x}}_{R}$ corresponding to $\left\{\mathbf{c}^{l}, \Delta \mathbf{c}^{l}\right\}$ and $p\left(\mathbf{c}^{l}, \Delta \mathbf{c}^{l}\right)$ is the joint probability of $\mathbf{c}^{l}$ and $\Delta \mathbf{c}^{l}$. Furthermore, $\|\cdot\|$ represents the Frobenius matrix norm. With the aid of the random interleaver $\boldsymbol{\Pi}_{R}(\cdot)$ at the RSs, the elements of $\mathbf{c}^{l}$ and $\Delta \mathbf{c}^{l}$ can be assumed to be mutually independent, respectively, and hence the joint probability $p\left(\mathbf{c}^{l}, \Delta \mathbf{c}^{l}\right)$ is written as

$$
p\left(\mathbf{c}^{l}, \Delta \mathbf{c}^{l}\right)=\prod_{i=1}^{K} p\left(\Delta c_{i}\right) p\left(c_{i}\right)
$$

Employing (5), the likelihood function of (4) is formulated as

$$
\begin{aligned}
& \sum_{\left\{\mathbf{c}^{l}, \Delta \mathbf{c}^{l}\right\}: c_{k}=1} e^{-\left\|\mathbf{r}_{B}-\mathbf{G} \tilde{\mathbf{x}}_{R}\right\|^{2} / \sigma^{2}} p\left(\mathbf{c}^{l}, \Delta \mathbf{c}^{l}\right) \\
& =\sum_{\left\{\mathbf{c}^{l}, \Delta \mathbf{c}^{l}\right\}: c_{k}=1} d\left(\tilde{\mathbf{x}}_{R}\right) \prod_{i=1}^{K} p\left(\Delta c_{i}\right) p\left(c_{i}\right),
\end{aligned}
$$

where $d\left(\tilde{\mathbf{x}}_{R}\right)=e^{-\left\|\mathbf{r}_{B}-\mathbf{G} \tilde{\mathbf{x}}_{R}\right\|^{2} / \sigma^{2}}$. Then, as in [14], we divide (6) into four terms with respect to the values of $c_{1}$ as well as
$\Delta c_{1}$ and combine them in order to obtain

$$
\begin{aligned}
& \sum_{\left\{\mathbf{c}^{l}, \Delta \mathbf{c}^{l}\right\}: c_{k}=1} d\left(\tilde{\mathbf{x}}_{R}\right) p\left(\mathbf{c}^{l}, \Delta \mathbf{c}^{l}\right) \\
= & \sum_{\left\{\mathbf{c}_{2}^{l}, \Delta \mathbf{c}_{2}^{l}, \hat{c}_{1}\right\}: c_{k}=1, \hat{c}_{1}=0} d\left(\tilde{\mathbf{x}}_{R}\right)\left\{\left(1-q_{1}\right) p\left(c_{1}=0\right)\right. \\
+ & \left.q_{1} p\left(c_{1}=1\right)\right\} \prod_{i=2}^{K} p\left(\Delta c_{i}\right) p\left(c_{i}\right) \\
+ & \sum_{\left\{\mathbf{c}_{2}^{l}, \Delta \mathbf{c}_{2}^{l}, \hat{c}_{1}\right\}: c_{k}=1, \hat{c}_{1}=1} d\left(\tilde{\mathbf{x}}_{R}\right)\left\{q_{1} p\left(c_{1}=0\right)\right. \\
+ & \left.\left(1-q_{1}\right) p\left(c_{1}=1\right)\right\} \prod_{i=2}^{K} p\left(\Delta c_{i}\right) p\left(c_{i}\right),
\end{aligned}
$$

where we have $\mathbf{c}_{i}^{l}=\left\{c_{i}, c_{i+1}, \cdots, c_{K}\right\}$ and $\Delta \mathbf{c}_{i}^{l}=$ $\left\{\Delta c_{i}, \Delta c_{i+1}, \cdots, c_{K}\right\}$. By using the a-priori probability of $\hat{c}_{i}$

$p\left(\hat{c}_{i}\right)= \begin{cases}\left(1-q_{i}\right) p\left(c_{i}=0\right)+q_{i} p\left(c_{i}=1\right), & \text { if } \hat{c}_{i}=0 \\ q_{i} p\left(c_{i}=0\right)+\left(1-q_{i}\right) p\left(c_{i}=1\right), & \text { if } \hat{c}_{i}=1\end{cases}$

we rewrite (7) as

$$
\begin{aligned}
& \sum_{\left\{\mathbf{c}^{l}, \Delta \mathbf{c}^{l}\right\}: c_{k}=1} d\left(\tilde{\mathbf{x}}_{R}\right) p\left(\mathbf{c}^{l}, \Delta \mathbf{c}^{l}\right) \\
= & \sum_{\left\{\mathbf{c}_{2}^{l}, \Delta \mathbf{c}_{2}^{l}, \hat{c}_{1}\right\}: c_{k}=1} d\left(\tilde{\mathbf{x}}_{R}\right) p\left(\hat{c}_{1}\right) \prod_{i=2}^{K} p\left(\Delta c_{i}\right) p\left(c_{i}\right) .
\end{aligned}
$$

Similar operations to (7) and (9) can be applied to the other coded bits $\left\{c_{2}, c_{3}, \cdots, c_{K}\right\}$ except for $c_{k}$ in order to obtain

$$
\begin{gathered}
\sum_{\left\{\mathbf{c}^{l}, \Delta \mathbf{c}^{l}\right\}: c_{k}=1} d\left(\tilde{\mathbf{x}}_{R}\right) p\left(\mathbf{c}^{l}, \Delta \mathbf{c}^{l}\right) \\
=p\left(c_{k}=1\right)\left\{\left(1-q_{k}\right) \sum_{\hat{\mathbf{c}}^{l}: \hat{c}_{k}=1} d\left(\tilde{\mathbf{x}}_{R}\right) \prod_{i=1, i \neq k}^{K} p\left(\hat{c}_{i}\right)\right. \\
\left.+q_{k} \sum_{\hat{\mathbf{c}}^{l}: \hat{c}_{k}=0} d\left(\tilde{\mathbf{x}}_{R}\right) \prod_{i=1, i \neq k}^{K} p\left(\hat{c}_{i}\right)\right\} .
\end{gathered}
$$

The likelihood function for $c_{k}=0$ can also be simplified by applying similar operations to those of (7), (9) and (10), yielding (11). Upon excluding the a-priori part, the extrinsic information is expressed as (12).

In [14], only a single RS having multiple antennas was employed for network-coding aided relaying systems. Therefore, the error rate $q_{k}$ is constant over the elements of $\hat{\mathbf{c}}^{l}$. However, in CSM systems employing multiple RSs, each RS independently decodes the received packet, a part of which is forwarded from each RS to the BS. Therefore, the parallel spatial substreams received at the BS have different BEPs $p_{n}$. In (12), it is seen that the extrinsic information of a coded bit is computed by using its specific error rate $q_{k}$. Furthermore, when $p\left(\hat{c}_{i}\right)$ is computed in (8), each coded bit's error probability $q_{i}$ is exploited. In order to employ the proposed decoding algorithm, the BEP information of $\left\{p_{1}, p_{2}, \cdots, p_{N_{R}}\right\}$ should be forwarded to the BS. However, if the length $L_{c}$ of the codeword is sufficiently high, the sideinformation transmission overhead of the BEP information is expected to modest. 


$$
L_{R}^{d, 1}\left(c_{k}\right)=\log \frac{p\left(c_{k}=1\right)}{p\left(c_{k}=0\right)}+\log \frac{\left(1-q_{k}\right) \sum_{\hat{\mathbf{c}}^{l}: \hat{c}_{k}=1} d\left(\tilde{\mathbf{x}}_{R}\right) \prod_{i=1, i \neq k}^{K} p\left(\hat{c}_{i}\right)+q_{k} \sum_{\hat{\mathbf{c}}^{l}: \hat{c}_{k}=0} d\left(\tilde{\mathbf{x}}_{R}\right) \prod_{i=1, i \neq k}^{K} p\left(\hat{c}_{i}\right)}{\left(1-q_{k}\right) \sum_{\hat{\mathbf{c}}^{l}: \hat{c}_{k}=0} d\left(\tilde{\mathbf{x}}_{R}\right) \prod_{i=1, i \neq k}^{K} p\left(\hat{c}_{i}\right)+q_{k} \sum_{\hat{\mathbf{c}}^{l}: \hat{c}_{k}=1} d\left(\tilde{\mathbf{x}}_{R}\right) \prod_{i=1, i \neq k}^{K} p\left(\hat{c}_{i}\right)} .
$$

$$
L_{R}^{e, 1}\left(c_{k}\right)=\log \frac{\left(1-q_{k}\right) \sum_{\hat{\mathbf{c}}^{l} l \hat{c}_{k}=1} d\left(\tilde{\mathbf{x}}_{R}\right) \prod_{i=1, i \neq k}^{K} p\left(\hat{c}_{i}\right)+q_{k} \sum_{\hat{\mathbf{c}}^{l}: \hat{c}_{k}=0} d\left(\tilde{\mathbf{x}}_{R}\right) \prod_{i=1, i \neq k}^{K} p\left(\hat{c}_{i}\right)}{\left(1-q_{k}\right) \sum_{\hat{\mathbf{c}}^{l}: \hat{c}_{k}=0} d\left(\tilde{\mathbf{x}}_{R}\right) \prod_{i=1, i \neq k}^{K} p\left(\hat{c}_{i}\right)+q_{k} \sum_{\hat{\mathbf{c}}^{l}: \hat{c}_{k}=1}^{K} d\left(\tilde{\mathbf{x}}_{R}\right) \prod_{i=1, i \neq k}^{K} p\left(\hat{c}_{i}\right)}
$$

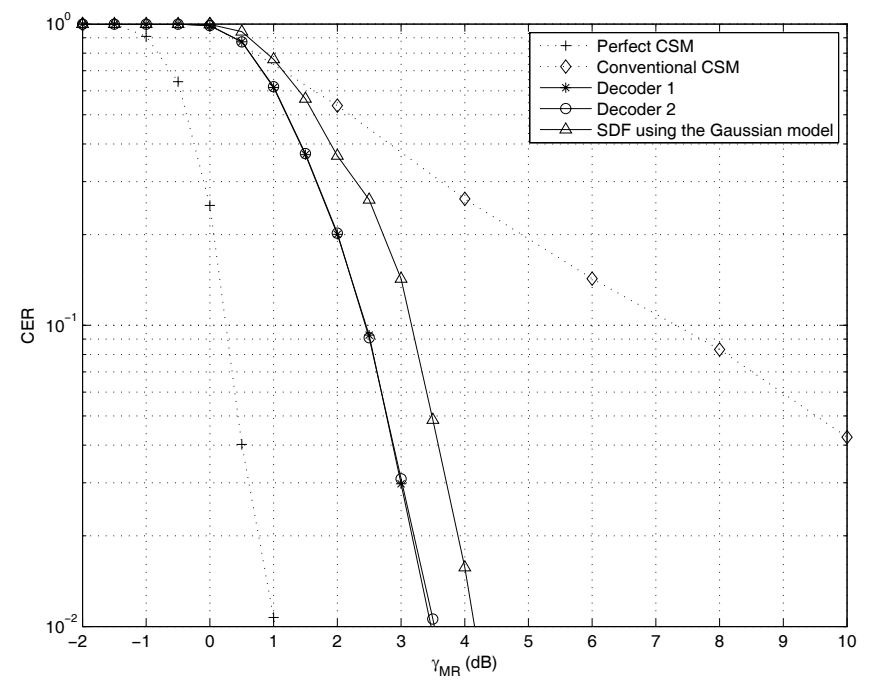

Fig. 3. CER versus SNR performance over a frequency-flat channel using $N_{R}=2, N_{B}=2, \gamma_{M R}=\gamma_{R B}, \gamma_{\Delta}=6 \mathrm{~dB}$.

\section{Simulation Results}

In order to evaluate the performance of the proposed decoding algorithm, we have performed computer simulations. In the MS-RS link as well as in the RS-BS link, we employed a turbo code having the rate $R=1 / 2$ and length of 1024 , which is constituted by two recursive systematic convolutional (RSC) codes with the octal generators $(7,5)$. Furthermore, QPSK signaling was used both in the MS-RS link as well as in the RS-BS link. The exact log-MAP channel decoding algorithm was adopted and the number of inner decoding iterations in the turbo channel decoder was set to five. In the IDD of the MIMO receiver, all possible symbol combinations are considered in the computation of (12), and the number of outer MIMO detection/decoding iterations was selected to be four.

All the elements of the MIMO channel matrices are assumed to be independent and identically distributed (i.i.d.) zero-mean complex Gaussian random variables having a variance of $1 / 2$ per dimension, which are constant in a TS. The SNR is defined as the ratio of the average power per information bit arriving at the receiver to the spectral density of the noise. The SNRs of the MS-RS and RS-BS links are denoted as $\gamma_{M R}$ and $\gamma_{R B}$, respectively. For simplicity, $\gamma_{M R}$ and $\gamma_{R B}$ are assumed to be the same throughout all the simulations, unless otherwise stated. The SNR of the direct link from the MS to the BS is denoted as $\gamma_{M B}$. It is also assumed that the relay channel has the same or a higher SNR compared to the direct channel (i.e. $\gamma_{M R} \geq \gamma_{M B}$ ) and we denote the ratio between these SNRs as $\gamma_{\Delta}(>1)$. In the RSBS link, the transmission power is equally distributed among multiple RSs, which implies that each RS transmits its signal at the same power.

In Figs. 3-5, where the codeword error ratio (CER) performance of various decoders is characterized, the perfect CSM denotes the idealized CSM scheme that transmits the perfectly decoded packets from the VAA, which do not suffer from decoding errors at the RSs. By contrast, the conventional CSM refers to the specific CSM scheme, which does not consider the potential presence of decoding errors at the RSs. Therefore, the BS assumes that perfectly decoded packets were transmitted form the RSs, regardless whether the forwarded packets actually contain hard-decision errors. In Decoder 1, namely the proposed scheme, the BS performs decoding by exploiting the algorithm described in Section III, while the perfect knowledge of the BEP $q_{k}$ is assumed. In Decoder 2, the BS also performs the decoding algorithm described in Section III, however the BEP $q_{k}$ is estimated with the aid of the algorithm described in [14]. The 'SDF using the Gaussian model' represents the soft-decision forwarding aided CSM system, where the expectation values of the decoded symbols are transmitted from the RSs in order to minimize the mean square error of the forwarded signal, while the soft-valued errors between the transmitted symbols and the exact values are assumed to be Gaussian random variables [15].

Fig. 3 illustrates the achievable CER performance of CSM systems using $N_{R}=2, N_{B}=2$, and $\gamma_{\Delta}=6 \mathrm{~dB}$. It is seen that Decoder 1 and Decoder 2 perform similarly, even though Decoder 2 does not assume the perfect knowledge of the BEP $q_{k}$. Furthermore, both Decoder 1 and Decoder 2 outperform the 'SDF using the Gaussian model' as well as the 'conventional CSM'. For a given received SNR of VAA, multiple RSs have different received power levels, which implies that some RSs can have substantially lower instantaneous SNRs than the others. The RSs having lower instantaneous SNRs may fail to decode the received packet, which results in erroneous forwarding even in the high-SNR region. In conventional CSM, the erroneous packets dominate the overall CER performance and hence the coding gain of the turbo code remains modest compared to the other decoders, as observed in Fig. 3.

In Fig. 4, the performance of CSM systems using $N_{R}=2$, $N_{B}=2$, and $\gamma_{\Delta}=18 \mathrm{~dB}$ is shown. In this scenario, the direct link has a much lower SNR than the relay link and hence the beneficial effect of the signal directly transmitted from the MS is almost negligible. In Fig. 4, it is observed that at $\mathrm{CER}=10^{-2}$, Decoder 1 provides an approximately 2.8 


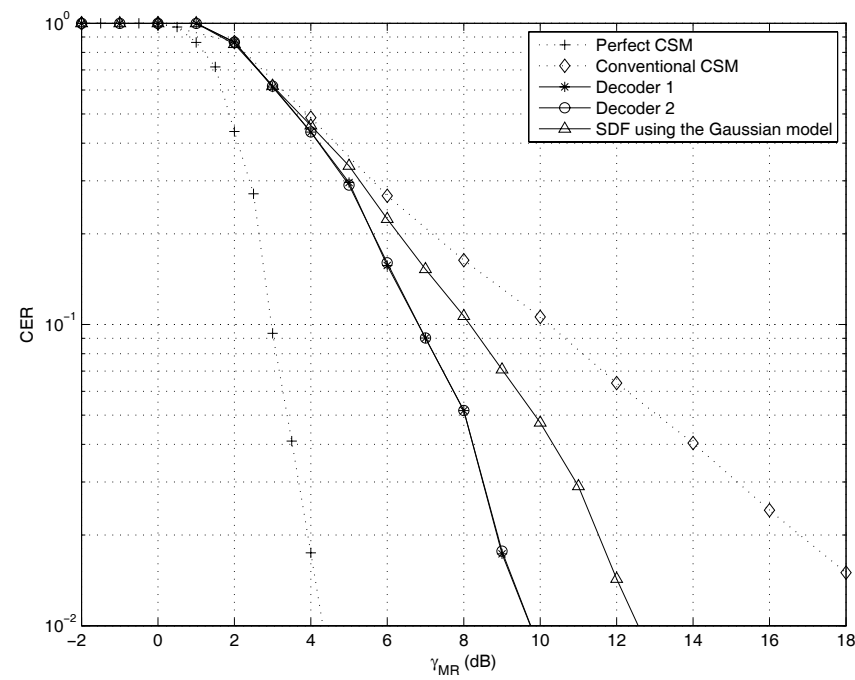

Fig. 4. CER versus SNR performance over a frequency-flat channel using $N_{R}=2, N_{B}=2, \gamma_{M R}=\gamma_{R B}, \gamma_{\Delta}=18 \mathrm{~dB}$.

dB SNR gain compared to the 'SDF using the Gaussian model'. Since the direct link is weak in this scenario, the accurate modeling of the relayed signal beneficially affects the achievable decoding performance at the BS. More specifically, the proposed algorithm of Section III employs a more accurate error model than the 'SDF using the Gaussian model' and hence it provides a substantial performance improvement, even though it exploits a simple hard-decision based forwarding scheme, rather than a soft-decision forwarding aided relaying scheme.

In Fig. 5, the performance of the $N_{R}=4, N_{B}=4$ and $\gamma_{\Delta}=18 \mathrm{~dB}$ scenario is characterised. Similar to Figs. 3-4, the proposed Decoders perform significantly better than the conventional CSM. Furthermore, they achieve an approximately $3.2 \mathrm{~dB}$ SNR gain over the 'SDF using the Gaussian model'. In Fig. 5, it is also observed that the proposed Decoders 1 and 2 have an approximately $3.1 \mathrm{~dB}$ SNR disadvantage with respect to the perfect CSM. This performance gap proves that the decoding errors at the RS significantly affect the overall system performance even though their effect is mitigated in the proposed Decoders 1 and 2.

We also performed simulations when $\gamma_{M R}$ is $9 \mathrm{~dB}$ higher than $\gamma_{R B}$, which implies that the RSs are considerably closer to the MS than to the BS. Fig. 6 characterizes the achievable CER performance of CSM systems using $N_{R}=4, N_{B}=4$, $\gamma_{\Delta}=18 \mathrm{~dB}$, and $\gamma_{R B}=\gamma_{M R}-9 \mathrm{~dB}$. In Fig. 6, the proposed Decoders 1 and 2 still achieve substantial SNR gains over the 'SDF using the Gaussian model' as well as over 'conventional $C S M$ ', even when the MS-RS link has a significantly better quality than the RS-BS link.

\section{CONCLUSION}

In this letter, the optimal decoding algorithm designed for hard-decision forwarding aided CSM systems has been derived. Since multiple RSs independently decode their received packets, which are forwarded to the BS, each RS may have a different decoding error rate. Therefore, in contrast to the decoding algorithm of [14], which employs a single RS in the

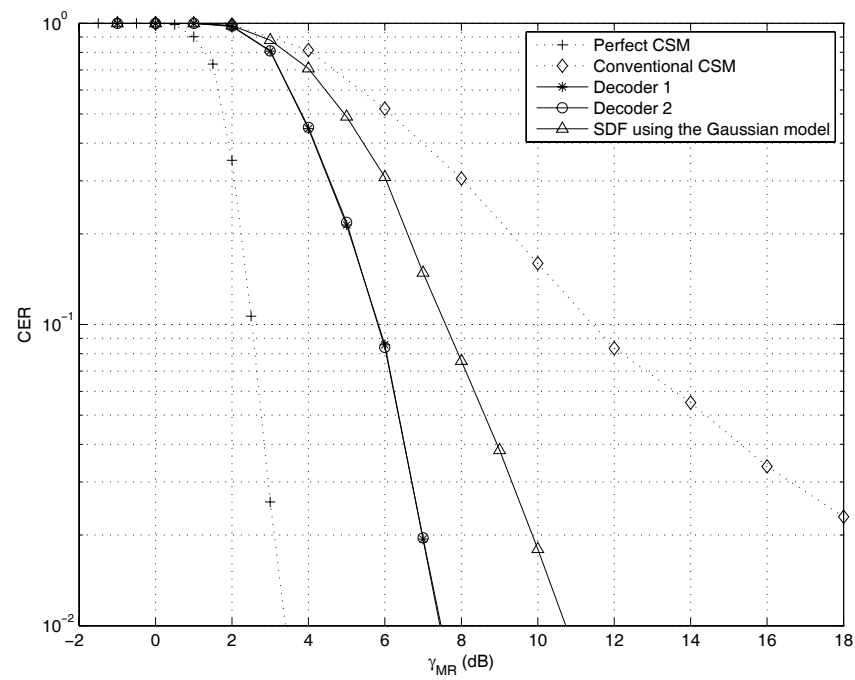

Fig. 5. CER versus SNR performance over a frequency-flat channel using $N_{R}=4, N_{B}=4, \gamma_{M R}=\gamma_{R B}, \gamma_{\Delta}=18 \mathrm{~dB}$.

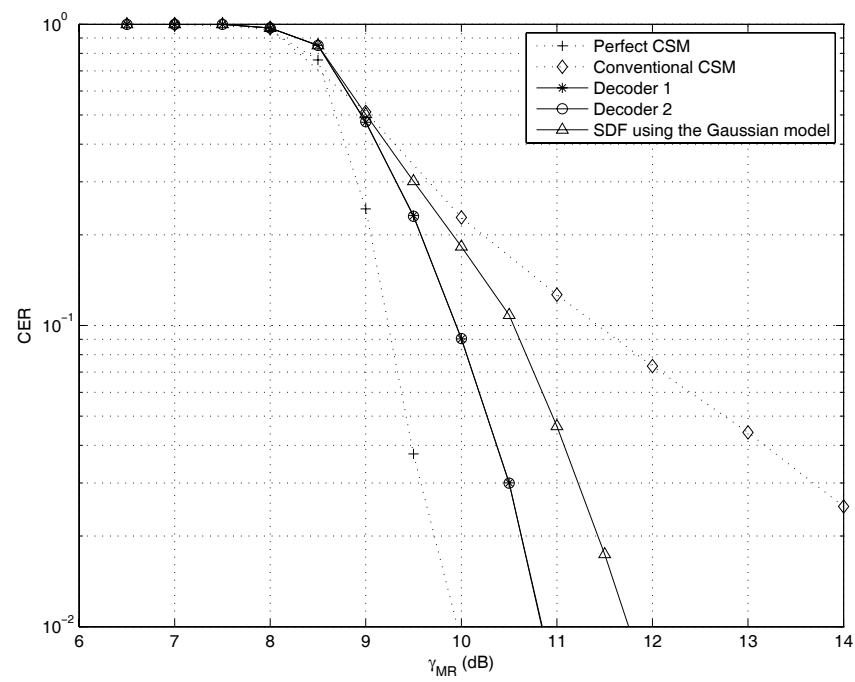

Fig. 6. CER versus SNR performance over a frequency-flat channel using $N_{R}=4, N_{B}=4, \gamma_{M R}=\gamma_{R B}+9 \mathrm{~dB}, \gamma_{\Delta}=18 \mathrm{~dB}$.

network-coding aided relaying system, the different BEP of each spatial stream is taken into account in order to create the optimal decoding algorithm for CSM systems. In the proposed algorithm, the erroneous forwarded signals can be corrected with the aid of the $\left(N_{R}-1\right)$ other spatial layer's signals as well as using the signal directly received from the MS. Our simulation results show that the proposed decoding algorithm designed for CSM systems provide substantial performance gains compared to the previously proposed algorithms, since the errors of the forwarded signals are taken into account with the aid of an accurate hard-valued error model. In order to achieve further improvements, the corresponding soft-decision forwarding strategy can be considered in our future work. By forwarding the soft-valued signals, which would more accurately indicate their reliability, the overall performance of CSM systems can be improved, provided that an accurate error model is employed. 


\section{REFERENCES}

[1] G. J. Foschini, "Layered space-time architecture for wireless communications in a fading environment when using multi-element antennas," Bell Labs Tech. J., vol. 1, pp. 41-59, 1996.

[2] G. J. Foschini and M. J. Gans, "On limits of wireless communications in a fading environment when using multiple antennas," Wireless Personal Commun., vol. 6, pp. 311-335, Mar. 1998.

[3] L. Hanzo, O. Alamri, M. El-Hajjar, N. Wu, Near-Capacity MultiFunctional MIMO Systems: Sphere-Packing, Iteratice Detection and Cooperation. IEEE Press - John Wiley, 2009

[4] J. N. Laneman and G. W. Wornell, "Energy-efficient antenna sharing and relaying for wireless networks," in Proc. IEEE Wireless Communications and Networking Conference, Chicago, IL, Sept. 2000, pp. 7-12.

[5] J. N. Laneman and G. W. Wornell, "Distributed space-time coded protocols for exploiting cooperative diversity in wireless networks," IEEE Trans. Inform. Theory, vol. 49, pp. 2415-2525, Oct. 2003.

[6] G. Scutari and S. Barbarossa, "Distributed space-time coding for regenerative relay networks," IEEE Trans. Wireless Commun., vol. 4, pp. $2387-$ 2399, Sept. 2005.

[7] M. El-Hajjar, S. Zummo, and L. Hanzo, "Near-instantaneously adaptive cooperative uplink schemes based on space-time block codes and VBLAST," in Proc. IEEE Vehicular Technology Conference, Dublin, Ireland, Apr. 2007, pp. 2200-2204.

[8] S. K. Jayaweera, "Virtual MIMO-based cooperative communication for energy-constrained wireless sensor networks," IEEE Trans. Wireless Commun., vol. 5, pp. 984-989, May 2006.
[9] Ö. Oyman and F. Xue, "Antenna selection, diversity coding and spatial multiplexing in cooperative wireless networks," in Proc. Asilomar Conference on Signals, Systems and Computers, Pacific Grove, CA, May 2007, pp. 814-818.

[10] S. W. Kim and R. Cherukuri, "Cooperative spatial multiplexing for high-rate wireless communications," in Proc. IEEE Workshop on Signal Processing Advances in Wireless Communications, New York, June 2005, pp. 181-185.

[11] Y. Zhang, G, Wang, and M. G. Amin, "Cooperative spatial multiplexing in multi-hop wireless networks," in Proc. IEEE International Conference on Acoustics, Speech and Signal Processing, Toulouse, France, May 2006, pp. 821-824.

[12] B. M. Hochwald and S. ten Brink, "Achieving near-capacity on a multiple-antenna channel," IEEE Trans. Inform. Theory, vol. 51, pp. 389 399, Mar. 2003.

[13] H. Vikalo, B. Hassibi, and T. Kailath, "Iterative decoding for MIMO channels via modified sphere decoding," IEEE Trans. Wireless Commun., vol. 3, pp. 2299-2311, Nov. 2004.

[14] K. Lee and L. Hanzo, "MIMO-aided hard versus soft decoding-andforwarding for network coding aided relaying systems," IEEE Trans. Wireless Commun., vol. 8, pp. 376-385, Jan 2009.

[15] Y. Li, B. Vucetic, T. F. Wong, and M. Dohler, "Distributed turbo coding with soft information relaying in multihop relay networks," IEEE $J$. Select. Areas Commun., vol. 24, pp. 2040-2050, Nov. 2006. 